\title{
Second International Cave Monitoring Workshop
}

\section{Innsbruck, Austria, 18-21 April 2012}

\section{David Mattey ${ }^{1}$, C. SpötL ${ }^{2}$ and M. Luetscher ${ }^{2}$}

'Department of Earth Sciences, University of London, UK; mattey@es.rhul.ac.uk

Institute of Geology and Palaeontology, University of Innsbruck, Austria

A successful paleoclimate reconstruction based on proxy archives rests not only on understanding how regional climate and local weather patterns are related to variability of the climate proxy, but equally on the understanding of local processes that influence how the climate signal is captured in the archive. Speleothem deposits in caves yield a wealth of proxy data that can be related to changes in atmospheric circulation, precipitation and vegetation.

Monitoring cave environments is key to understanding relationships between weather and the processes that affect calcite precipitation inside caves. These include for example knowledge of the production rate of $\mathrm{CO}_{2}$ in the soil zone and its movement in and out of caves, the relationship between rainfall and the composition and movement of groundwater, and the impact of seasonal changes on the precipitation of carbonate as speleothem. Setting up and maintaining reliable cave monitoring programs presents a challenge in wet caves, which are often remote and difficult to access even for experienced speleologists.
Back in 2009 we held a first workshop on cave monitoring techniques in Gibraltar to discuss the technology that was available and share experiences in best practices among the small group of researchers in this field. Three years later many more groups are involved in cave monitoring and have acquired considerable expertise. In April 2012 we held the $2^{\text {nd }}$ International Cave Monitoring Workshop in Innsbruck bringing together scientists and PhD students from 14 countries to discuss new ideas in methodology and instrumentation.

The workshop provided an informal discussion forum for exchange of experiences covering all practical aspects of cave monitoring. Four main themes were discussed: strategies for monitoring the hydrology and meteorology of cave systems, instrumentation and data logging, sampling and analysis protocols, and insitu studies of carbonate precipitation and other cave processes. It is clear that some challenges still remain, such as finding a reliable way to precisely measure the relative humidity, and avoiding unwanted interference by insects and other animals as well as humans. However, the three years since the first workshop saw significant advances in the ability to carry out automatic logging and sampling of key parameters using ingenious, custom-built instruments.

Cave monitoring is now being actively carried out in many cave systems over the world (e.g. Fig 1) and clearly shows that every cave has its own "personality" that takes several annual cycles to properly characterize and evaluate. Once this is done, features of the chemical proxy record preserved in speleothem can then be assigned to specific local processes, and thus improve the reliability of climate reconstructions from proxy records.

A day trip to Spannagel Cave, an 11 $\mathrm{km}$-long high-alpine marble cave hosting Europe's highest show cave, marked the end of the workshop. The workshop organizers are grateful for financial support from the Office of the Vice Rector for Research, the Faculty of Geo- and Atmospheric Sciences and the Research Centre Geodynamics and Geomaterials (all University of Innsbruck, Austria) and Gemini Data Loggers (UK).

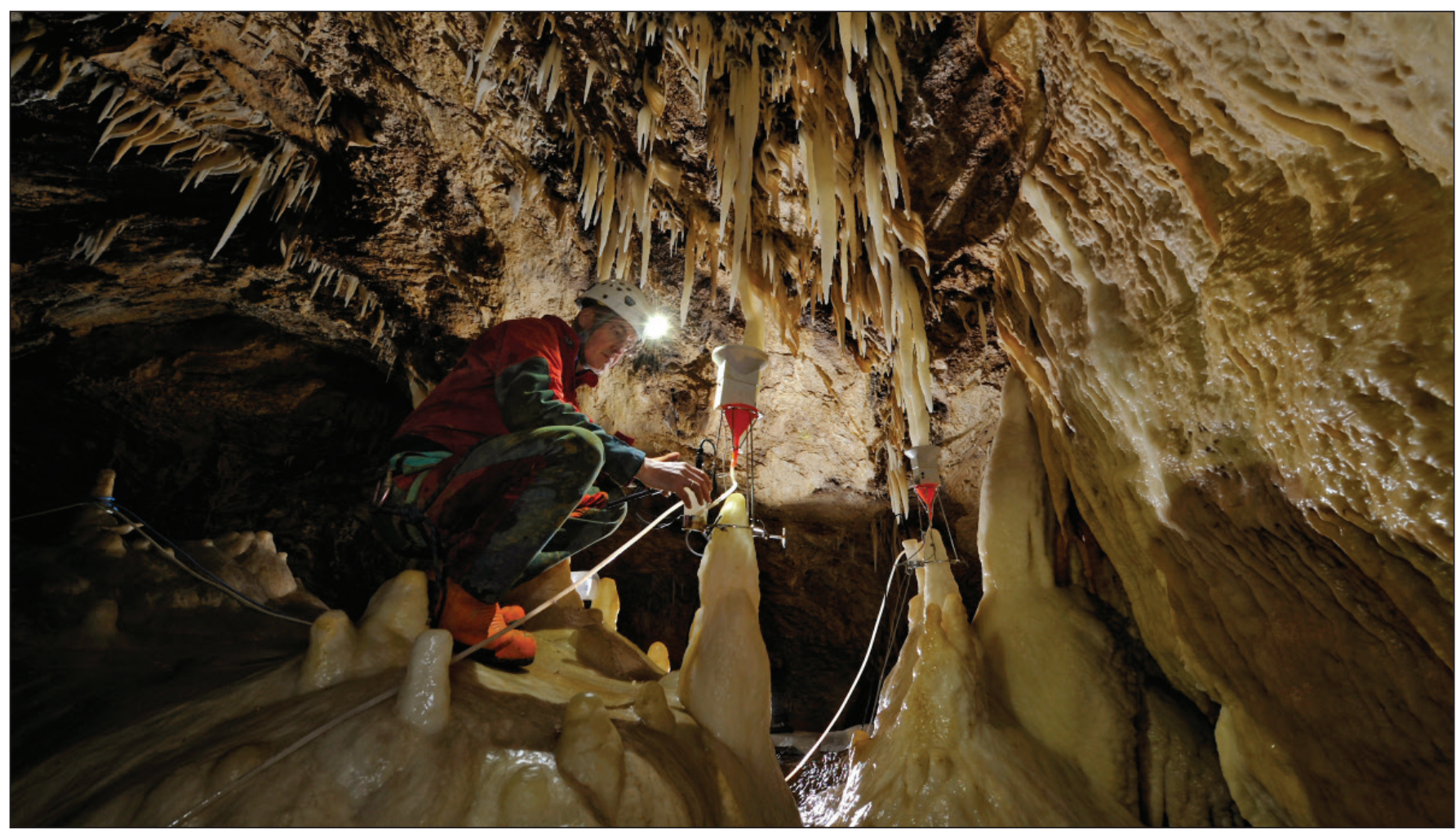

Figure 1: Dripwater collection in Obir Cave, Austria. Photo credit: Robbie Shone (shonephotography.com). 